\title{
Effect of Aortic Stent Implantation in the Treatment of Thoracic Aortic Disease
}

\author{
Cheng Chen \\ Vascular Surgery, YUNNAN FUWAI CARDIOVASCULAR HOSPITAL, Kunming, Yunnan, 650000, China
}

\begin{abstract}
[Abstract] Objective: To explore the effect of aortic stent implantation on patients in the treatment of thoracic aortic diseases. Methods: Selected patients from Yunnan Fuwai Cardiovascular (YFC) Hospital as a sample group to carry out the study, the main participants were thoracic aortic aneurysm patients admitted from June 2020 to June 2021. The number of patients involved were 80. The patients are divided into two groups and different treatment methods were adopted. A comparative analysis of the effects of aortic stent placement on patients was conducted. Results: Before treatment, there was no significant difference in the levels of various inflammatory factors between the two groups of patients, $P>0.05$. After treatment, the data indicators of the two groups were significantly different, as there were significant differences in the surgical indicators among the two groups of patients, $\mathrm{P}<0.05$. As a result, the experimental group had shorter operation time, less intraoperative blood loss, shorter ICU observation time, lower levels of inflammatory factors, and better results. Conclusion: In the treatment of patients with thoracic aortic disease, the application of aortic stent implantation has significant clinical effects and are more conducive to the recovery of patients. It can be promoted and used in clinical trials.
\end{abstract}

Keywords: Aortic stent placement; Thoracic aortic aneurysm; Clinical effect

Publication date: May, 2021; Publication online: 31 May, 2021

*Corresponding author: Cheng Chen, 13578086160@sina.cn

\section{Introduction}

There are many types of thoracic aortic diseases, including aortic dissection, aortic aneurysm, traumatic aortic rupture, etc., which are highly life threatening to the health of the patient. When the patient is treated with surgery alone, the patient is at greater risk and is not conducive to the patient recovery ${ }^{[1]}$. In recent years, stent technology has gradually developed and has been applied in patients with thoracic aortic disease. This method is not only simple in operation technology, but can also reduce the incidence of post-operative complications in patients, as well as have a high clinical application value ${ }^{[2]}$. This study is based on, selecting patients with thoracic aortic aneurysm as a sample, and implanting aortic stent technology to explore its clinical application effects.

\section{Data analysis and methods}

\subsection{General information}

In this study, 80 patients with thoracic aortic aneurysm from
YFC hospital were selected as the research sample, and the study was carried out from June 2020 to June 2021. Among them, there were 45 male patients and 35 female patients. The age of the patients was selected at the range of 40-75 years old, and the mean was $(56.45 \pm 3.21)$ years old. In this study, patients were divided into two groups with different treatment methods. The names of the groups were the experimental group $(n=40)$ and the control group $(n=40)$. The general data of the two groups of patients were compared and analyzed. Under the principle of equal distribution, the differences in various indicators were small, $\mathrm{P}>0.05$, which were compared between groups.

\subsection{Research methods}

\subsubsection{Control group (n=40): Artificial blood vessel} replacement surgery

During the specific implementation process, the patient was given anesthesia intervention, followed by a selected surgical incision on the patient's chest, then the patient's condition was observed, his brachiocephalic artery and right 
subclavian artery were freed. Then, the right subclavian artery was selected for intubation, and a stepped tube was entered into the atrium for relaxation to maintain an effective extracorporeal circulation system for the patient. Upon that, cooling treatment was given for temperature control; mainly to control the degree of cardiac arrest. Following that, the patient was given a frontal aortic arch replacement and an implantation of the stent trunk. This completed the blood vessel anastomosis. Finally, full revival procedure was performed, where the aortic blocking clamp was opened, and after the patient resumes the spontaneous heartbeat, "pump and re-beat" was done to complete the operation. This procedure was done for all 40 patients of this sample group.

\subsubsection{Experimental group $(n=40)$ : Intraluminal stent} graft implantation

First the patient was given anesthesia intervention, and then the patient was implanted with a catheter after the brachial artery or femoral artery punctured. The implantation location was at the ascending aorta, and the patient was given angiographic intervention to facilitate the observation, to understand the situation and clarify the blood supply of the patient's aorta and in the brachiocephalic blood vessels. Then evaluation of whether the patient could undergo endovascular surgery was done, and the distortion, occlusion and stenosis of the patient was assessed. If the patient could undergo endovascular surgery, it was necessary to externally cut the femoral artery at the lower abdomen, to use it as the entrance port, and choose an artificial stent for the blood vessel. The stent was then placed using a guide wire into the patient's aorta, according to the actual positioning. The artificial blood vessel of the stent was released and the patient was given another imaging. The conditions after implantation was observed to determine the sealing of the aneurysm cavity. If necessary, the arterial stent can be inserted into the patient's left common carotid artery and in the left subclavian artery. The operation was subsequently completed. This procedure was done for all 40 patients of this sample group.

\subsection{Observation indicators}

Observed the patient's operation status, including operation time, intraoperative blood loss and ICU observation time, etc., and compared the levels of inflammatory factors before and after the operation.

\subsection{Statistical methods}

For data statistics, the statistical software mainly used was SPSS20.0, and the statistical data mainly included measurement data, which is expressed by ( $\left.x_{ \pm \mathrm{s}}\right)$ and verified, and the result was verified by the $t$ value. There is significance when $\mathrm{P}<0.05$.

\section{Results}

\subsection{Comparison of surgical indications}

There were significant differences in the operation time, intraoperative blood loss and ICU observation indexes between the two groups of patients, which is expressed as $\mathrm{P}<0.05$, and the experimental group has better results, as shown in Table 1.

Table 1. Comparison of surgical indications between the two groups ( $x_{ \pm \mathrm{s}}$ )

\begin{tabular}{cccc}
\hline Group & Operation time $(\mathbf{m i n})$ & Intraoperative blood loss (m) & ICU observation time (h) \\
\hline Test group $(\mathrm{n}=40)$ & $151.23 \pm 32.34$ & $60.54 \pm 25.43$ & $9.23 \pm 2.11$ \\
Control group $(\mathrm{n}=40)$ & $226.57 \pm 46.50$ & $965.67 \pm 76.55$ & $35.49 \pm 5.46$ \\
$\mathrm{t}$ & 8.495 & 35.493 & 6.500 \\
$\mathrm{P}$ & $<0.05$ & $<0.05$ & $<0.05$ \\
\hline
\end{tabular}

\subsection{Comparison of inflammatory factor levels}

\section{before and after surgery}

Before treatment, there was no significant difference in the levels of various inflammatory factors between the two groups of patients, $\mathrm{P}>0.05$. After treatment, the indicators of the two groups of patients changed, which were significantly different from those before treatment, $\mathrm{P}<0.05$. At the same time, after treatment, the data indicators of the two groups are quite different, $\mathrm{P}<0.05$, as shown in Table 2 .

Table 2. Comparison of the levels of inflammatory factors before and after surgery between the two groups ( $x_{ \pm \mathrm{s}}$ ) 


\begin{tabular}{ccccccc}
\hline \multirow{2}{*}{ Group } & \multicolumn{2}{c}{ IL-6 $(\mathbf{n g} / \mathbf{L})$} & \multicolumn{2}{c}{$\mathbf{C R P}(\mathbf{m g} / \mathbf{L})$} & \multicolumn{2}{c}{ TNF- $\boldsymbol{\alpha}(\mathbf{P G} / \mathbf{m l})$} \\
\cline { 2 - 6 } & $\begin{array}{c}\text { Before } \\
\text { treatment }\end{array}$ & After treatment & $\begin{array}{c}\text { Before } \\
\text { treatment }\end{array}$ & After treatment & $\begin{array}{c}\text { Before } \\
\text { treatment }\end{array}$ & After treatment \\
\hline $\begin{array}{c}\text { Test group } \\
(\mathrm{n}=40)\end{array}$ & $15.45 \pm 3.22$ & $34.56 \pm 4.21$ & $6.23 \pm 1.01$ & $13.24 \pm 2.87$ & $18.76 \pm 8.13$ & $31.57 \pm 7.98$ \\
$\begin{array}{c}\text { Control group } \\
(\mathrm{n}=40)\end{array}$ & $15.45 \pm 2.56$ & $66.75 \pm 5.32$ & $6.34 \pm 0.87$ & $31.24 \pm 3.65$ & $18.76 \pm 8.65$ & $51.24 \pm 10.76$ \\
$\mathrm{t}$ & 0.453 & 6.553 & 0.394 & 8.792 & 0.284 & 6.321 \\
$\mathrm{P}$ & $>0.05$ & $<0.05$ & $>0.05$ & $<0.05$ & $>0.05$ & $<0.05$ \\
\hline
\end{tabular}

\section{Discussion}

In recent development, stent placement technology has a wide range of applications in thoracic aortic aneurysm disease. Patients with thoracic aortic disease are critically ill, and when general surgery is used, the risk is greater, therefore, in clinical trials, the applicability is low ${ }^{[3]}$. The stent cavity isolation technology has the same indications as surgical indications, especially in older patients with thoracic aortic disease. The combination of stent placement and surgery can promote a wider clinical application of stents. When compared with traditional surgical methods, this technology can reduce the surgical trauma of patients and has a positive significance for patient recovery ${ }^{[4]}$. In this study, for patients with thoracic aneurysms, the intraluminal stent graft placement in the aortic stent treatment technology was applied, this technology is to apply the stent-indicated stent graft to cover the patient's aneurysm distal and proximal ends, reduce the patient's tumor cavity pressure, while promoting its internal blood flow ${ }^{[5]}$. Moreover, the components of the covered stent include platinum and the outer membrane is polytetrafluoroethylene, which can promote the expansion strength of the stent, reduce the incidence of vascular restenosis, avoid the patient's vascular injury, and reduce the incidence of complications. Rate of clinical application significance is significant ${ }^{[6]}$. After applying this technique to patients with thoracic aneurysm, the results showed that the experimental group had shorter operation time, less blood loss, and lower levels of inflammatory factors after treatment, which were significantly different from those in the control group, where it is expressed as $\mathrm{P}<0.05$, indicating that the application effect of aortic stent treatment technology is significant.

\section{Conclusion}

In summary, the application of aortic stent implantation for patients with thoracic aortic aneurysm have significant clinical effects. It can improve the level of inflammation in patients, and have positive significance for clinical development and can be recommended to patients.

\section{References}

[1] Li JR, Xu SD, Zheng J, et al., 2020, Efficacy of Aortic Endovascular Repair and Intraoperative Stent Grafting in the Treatment of Traumatic Descending Aortic Arch Lesions, Journal of Practical Medicine. (19).

[2] Tang YF, Han L, Fan XL, et al., 2019, Analysis of Surgical Treatment of Stent Infection after Endovascular Repair of Thoracic Aorta, Chinese Journal of Surgery. 57(011):848-852.

[3] Cui WC, Zhang JQ., 2020, Analysis of the Application Effect of Endovascular Stent Grafting in the Treatment of Thoracic Aortic Aneurysms, Sichuan Journal of Anatomy. 28(02):176-177.

[4] Mu S, Zhang HM, Zhang LX, et al., 2019, Application and Numerical Analysis of a New Type of Thoracic-Abdominal Aortic Stent Graft in the Treatment of Arterial Diseases, China Water Transport (second half of the month). 19(004):95- 97.

[5] Li XL, He P, Zhang DY, et al., 2020, Clinical Analysis of 3D Printing-Assisted Endovascular Repair of Complex Thoracic Aortic Vascular Disease Branch Stent, Chinese Journal of Thoracic and Cardiovascular Surgery. 27(02):113-117.

[6] Ning Y, Chen J, Feng Z., 2019, The Effect of Endovascular Stent Grafting for Thoracoabdominal Aortic Aneurysm on Intraoperative Blood Loss and Postoperative Recovery, Youjiang Medical Science. 